\title{
Analysis of Inherent Irreversibility in a Variable Viscosity MHD Generalized Couette Flow with Permeable Walls*
}

\author{
Oluwole Daniel MAKINDE** and Adetayo Samuel EEGUNJOBI*** \\ ${ }^{* *}$ Institute for Advanced Research in Mathematical Modelling and Computation, Cape Peninsula, University \\ of Technology, P.O.Box 1906, Bellville 7535, South Africa. \\ ***Department of Mathematics and Statistics, Polytechnic of Namibia (Namibia's University of Science and \\ Technology), Private Bag 13388, 13 Storch Street, Windhoek, Namibia. \\ E-mail: samdet1@yahoo.com
}

\begin{abstract}
The inherent irreversibility in a variable viscosity hydromagnetic generalized Couette flow with suction/injection at the walls has been investigated theoretically. Using a fourth-order Runge-Kutta-Fehlberg integration scheme together with shooting technique, the model equations for momentum and energy balance are tackled numerically. The velocity and the temperature profiles are obtained and are utilized to compute the skin friction coefficient, Nusselt number, entropy generation rate and the Bejan number. The results are presented graphically and discussed quantitatively for several values of thermophysical parameters controlling the flow regime. Our results reveal that a decrease in fluid viscosity enhances dominant effect of heat transfer irreversibility and the imposition of magnetic field damping the entropy generation rate in the flow system.
\end{abstract}

Key words: Generalized MHD Couette Flow, Variable Viscosity, Suction/Injection, Heat Transfer, Entropy Generation, Bejan Number

\section{Introduction}

Magnetohydrodynamic (MHD) phenomena are outcome of mutual interaction between magnetic field and electrically conducting fluid flowing across it. Interest in the theoretical study of hydromagnetic flow of an electrically conducting fluid and heat transfer between two parallel plates, one of which is moving relative to the other known as Couette flow is motivated by several important problems in engineering and industries. Hydromagnetic principles is used to design heat exchangers, pumps, accelerators and flow-meters, in solving space vehicle propulsion, control and re-entry problems, in creating novel electric current generating systems and in developing confinement schemes for controlled nuclear fusion [1]. Agarwal [2] studied the problem of generalized Couette flow under a transverse magnetic field and obtained the conditions for back-flow as well as results for viscous drag at the stationary plate. The heat transfer aspect of MHD generalized Couette flow has been studied by Soundalgekar [3]. Thereafter, many researchers have investigated the velocity profiles and heat transfer rate in MHD flow under various physical situation [4-6]. Makinde and Onyejekwe [7] reported a numerical solution for MHD generalized Couette flow

Received 18 Jan., 2013 (No. 13-0015) [DOI: 10.1299/jtst.8.240]

Copyright () 2013 by JSME and heat transfer with variable viscosity and electrical conductivity. 
Meanwhile, entropy generation analysis has become an important aspect of modelling and optimizing application in fluid flow and energy system to find their optimum design condition. This enables us to identify the factors that are major cause of irreversibility effects in order to reduce the entropy production and improve the system efficiency. In a pioneering work, Bejan [8] presented a theoretical analysis of entropy generation in flow systems and heat transfer processes. Mahmud and Fraser [9] studied the entropy generation characteristic inside a porous channel with viscous dissipation. Aziz [10] investigated the entropy generation in Couette flow assisted with pressure gradient and considered four different combinations of thermal boundary conditions. Eegunjobi and Makinde [11] studied the effect of Navier slip on entropy generation in a porous channel with suction/injection. Suction/injection plays an important role in the control of flow past an infinite permeable plate or within parallel permeable plates, hence its importance in practical problems involving film cooling, control of boundary layers in industrial, geophysical, biomedical, engineering and environmental applications. Shojaefard et al.[12] investigated flow control on a subsonic airfoil by suction/injection and concluded that suction significantly increases the lift coefficient while injection decreases the surface skin friction, which transitively resulted in a considerable reduction in energy consumed during flights of subsonic aircrafts. Other researchers such as Makinde and Eegunjobi [13], Wood [14], Tasnim et al. [15], etc., have also investigated entropy generation rate in a thermal system under various situations. From the literature survey, it seems that the problem of entropy generation in a variable viscosity generalized MHD Couette flow with permeable boundaries has not received much attention.

In the work reported herein, the combined effects of variable viscosity and suction/injection on entropy generation rate in a generalized hydromagnetic Couette flow with permeable boundaries are investigated. In the following sections, the model problem is obtained and solved numerically using a shooting method coupled with a fourth-order Runge-Kutta-Fehlberg integration scheme. Pertinent results are presented graphically and discussed quantitatively.

\section{Mathematical Model}

We consider a steady, incompressible, laminar flow of an electrically conducting variable viscosity fluid between a fixed permeable lower plate and a moving permeable upper plate. The fluid is acted upon by a constant pressure gradient and an external uniform magnetic field is applied perpendicular to the plates as illustrated in figure 1. It is assumed that the fluid is injected uniformly into the channel at the lower plate while the uniform fluid suction occurs at the moving upper plate. A transverse magnetic field with strength $B_{0}$ is applied parallel to the $y$-axis. There is no applied voltage and the magnetic Reynolds number is small, hence the induced magnetic field and Hall effects are negligible. 


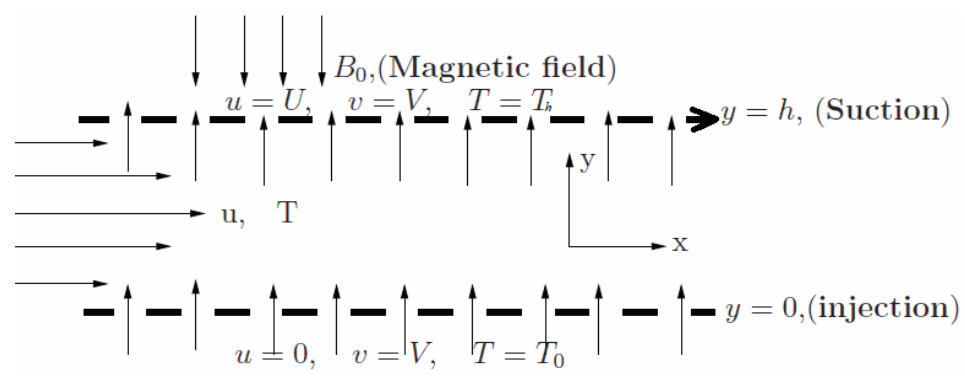

Figure 1: Schematic diagram of the problem

Under these assumptions, the governing equations for the momentum and energy balance in one dimension can be written as follows $[7,9,10,13]$

$$
V \frac{d u}{d y}=-\frac{1}{\rho} \frac{d P}{d x}+\frac{1}{\rho} \frac{d}{d y}\left(\bar{\mu}(T) \frac{d u}{d y}\right)-\frac{\sigma B_{0}^{2} u}{\rho}
$$

and

$$
V \frac{d T}{d y}=\alpha \frac{d^{2} T}{d y^{2}}+\frac{\bar{\mu}(T)}{\rho c_{P}}\left(\frac{d u}{d y}\right)^{2}+\frac{\sigma B_{0}^{2} u^{2}}{\rho c_{p}} .
$$

The boundary conditions are

$$
\begin{aligned}
& u(0)=0, T(0)=T_{0}, \\
& u(h)=U, T(h)=T_{h},
\end{aligned}
$$

where $h$ is the channel width, $u$ is the velocity of the fluid, $P$ is the fluid pressure, $V$ is the uniform suction / injection velocity at the channel walls, $U$ is the uniform velocity of the upper wall, $\alpha$ is the thermal diffusivity, $\rho$ is the fluid density, $\sigma$ is the fluid electrical conductivity, $k$ is the thermal conductivity coefficient, $c_{p}$ is the specific heat at constant pressure, $T$ is the fluid temperature, $T_{0}$ is the lower stationary wall temperature and $T_{0}$ is the upper wall temperature. The temperature dependent viscosity $\bar{\mu}$ can be expressed as [16]

$$
\bar{\mu}(T)=\mu_{0} e^{-m\left(T-T_{0}\right)},
$$

where $m$ is a viscosity variation parameter and $\mu_{0}$ is the fluid dynamic viscosity at lower fixed wall. Using (1) and (3b), the constant axial pressure gradient will be given as

$$
-\frac{1}{\rho} \frac{d p}{d x}=\frac{\sigma B_{0}^{2} U}{\rho},
$$

and equations (1) and (2) then become

$$
V \frac{d u}{d y}=\frac{1}{\rho} \frac{d}{d y}\left(\bar{\mu}(T) \frac{d u}{d y}\right)-\frac{\sigma B_{0}^{2}(u-U)}{\rho},
$$




$$
V \frac{d T}{d y}=\alpha \frac{d^{2} T}{d y^{2}}+\frac{\bar{\mu}(T)}{\rho c_{P}}\left(\frac{d u}{d y}\right)^{2}+\frac{\sigma B_{0}^{2}}{\rho c_{p}}(u-U)^{2} .
$$

We introduce the following non-dimensional quantities:

$$
\eta=\frac{y}{h}, \alpha=\frac{k}{\rho c_{p}}, w=\frac{u}{U}, \theta=\frac{T-T_{0}}{T_{h}-T_{0}}, \bar{P}=\frac{p h}{\mu_{0} U}, \mu=\frac{\bar{\mu}}{\mu_{0}}, v=\frac{\mu_{0}}{\rho} \text {. }
$$

Substituting equation (8) into equations (6)-(7), we obtain

$$
\begin{aligned}
& \frac{d^{2} w}{d \eta^{2}}-\varepsilon \frac{d \theta}{d \eta} \frac{d w}{d \eta}-e^{\varepsilon \theta}\left(\operatorname{Re} \frac{d w}{d \eta}+H a(w-1)\right)=0, \\
& \frac{d^{2} \theta}{d \eta^{2}}-\operatorname{Re} \operatorname{Pr} \frac{d \theta}{d \eta}+E c \operatorname{Pr} e^{-\varepsilon \theta}\left(\frac{d w}{d \eta}\right)^{2}+E c \operatorname{Pr} H a(w-1)^{2}=0,
\end{aligned}
$$

with the boundary conditions

$$
\left.\begin{array}{cc}
w(0)=0, & \theta(0)=0, \\
w(1)=1, & \theta(1)=1,
\end{array}\right\}
$$

where

$\operatorname{Re}=\frac{V h}{v}($ Reynolds number $), \quad \operatorname{Pr}=\frac{v}{\alpha}($ Prandtl number $)$,

$E c=\frac{U^{2}}{c_{p}\left(T_{h}-T_{0}\right)}$ (Eckert number), $H a=\frac{\sigma B_{0}^{2} h^{2}}{\mu_{0}}$ (Magnetic field parameter),

$\varepsilon=m\left(T_{h}-T_{0}\right) \quad($ Viscosity exponent $)$.

It is important to note that $\varepsilon=0$ corresponds to the case of constant viscosity conducting fluid. The exact solution of equation (9) for the fluid velocity is possible under this constant viscosity scenario and we obtain

$$
w(\eta)=1+\frac{e^{a \eta+b}-e^{b \eta+a}}{e^{a}-e^{b}},
$$

where $a=\left(\operatorname{Re}+\sqrt{\operatorname{Re}^{2}+4 H a}\right) / 2$ and $b=\left(\operatorname{Re}-\sqrt{\operatorname{Re}^{2}+4 H a}\right) / 2$.

Other important physical quantities of interest in this problem are the skin friction coefficient $C_{f}$ and Nusselt number $N u$ which are defined as:

$$
C_{f}=\frac{h \tau_{w}}{\mu_{0} U}, \quad N u=\frac{q_{w} h}{k\left(T_{h}-T_{0}\right)},
$$

where the skin friction $\tau_{w}$, heat flux $q_{w}$ at the channel walls are given by

$$
\tau_{w}=\left.\bar{\mu}(T) \frac{\partial u}{\partial y}\right|_{y=0,1}, \quad q_{w}=-\left.k \frac{\partial T}{\partial y}\right|_{y=0,1} .
$$

Substituting equation (14) into (13), we obtain

$$
C_{f}=\left.e^{-\varepsilon \theta} \frac{d w}{d \eta}\right|_{\eta=0,1}, \quad N u=\left.\frac{d \theta}{d \eta}\right|_{\eta=0,1} .
$$




\section{Entropy Analysis}

Magnetohydrodynamic and heat transfer processes in a generalized Couette flows with permeable boundaries are irreversible. This inherent irreversibility arises due to the exchange of energy and momentum within the fluid and at permeable boundaries, thus resulting in entropy generation. Following Wood [14], the local volumetric rate of entropy generation $E_{G}$ for a viscous incompressible conducting fluid in the presence of magnetic field is defined by

$$
E_{G}=\frac{k}{T_{0}^{2}}\left(\frac{d T}{d y}\right)^{2}+\frac{\mu}{T_{0}}\left(\frac{d u}{d y}\right)^{2}+\frac{\sigma B_{0}^{2}}{T_{0}}(u-U)^{2} .
$$

The first term in equation (16) is irreversibility due to heat transfer, the second term is entropy generation due to viscous dissipation and the third term is local entropy generation due to the effect of the Joule dissipation. Substituting equation (8) into equation (16), we obtain the dimensionless form of local entropy generation rate as

$$
N s=\frac{T_{0}^{2} h^{2} E_{G}}{k\left(T_{h}-T_{0}\right)^{2}}=\left(\frac{d \theta}{d \eta}\right)^{2}+\frac{B r}{\Omega}\left[e^{-\varepsilon \theta}\left(\frac{d w}{d \eta}\right)^{2}+H a(w-1)^{2}\right],
$$

where $\Omega=\left(T_{h}-T_{0}\right) / T_{0}$ is the temperature difference parameter and $B r=E c P r$ is the Brinkman number. The Bejan number Be is define as

$$
B e=\frac{N_{1}}{N_{s}}=\frac{1}{1+\Phi},
$$

where Ns $=N_{1}+N_{2}$,

$N_{1}=\left(\frac{d \theta}{d \eta}\right)^{2}$ (Heat transfer irreversibility),

$N_{2}=\frac{B r}{\Omega}\left[e^{-\varepsilon \theta}\left(\frac{d w}{d \eta}\right)^{2}+H a(w-1)^{2}\right]$ (Fluid friction and magnetic field irreversibility),

$\Phi=\frac{N_{2}}{N_{1}}$ (Irreversibility ratio).

Equation (18) shows that Bejan number ranges from 0 to $1 . B e=0$ is the limit where the irreversibility is dominated by the combined effects of fluid friction and magnetic fields and $B e=1$ is the limit where the irreversibility due to heat transfer dominates the flow system by virtue of finite temperature differences.

\section{Numerical Procedure}

The coupled nonlinear boundary value problem represented by equations (9)-(10) together with their boundary conditions in equation (11) have been solved numerically using an efficient fourth-order Runge-Kutta-Fehlberg integration scheme 
together with shooting technique [17]. The basic idea of the shooting method for solving boundary value problem is to try to find the appropriate initial condition for which the computed solution hits the target so that the boundary conditions at the other points are satisfied.

Firstly, the model nonlinear boundary value problem in equations (9)-(10) together with the conditions (11) is reduced to a system of initial value problem. Let

$$
w=x_{1}, w^{\prime}=x_{2}, \theta=x_{3}, \theta^{\prime}=x_{4},
$$

where the prime symbol denotes the derivative with respect to $\eta$. Substituting equation (19) into equations (9)-(11), we obtain

$$
\begin{aligned}
& x_{1}^{\prime}=x_{2}, \\
& x_{2}^{\prime}=\varepsilon x_{2} x_{4}+e^{\varepsilon x_{3}}\left(\operatorname{Re} x_{2}+H a\left(x_{1}-1\right)\right), \\
& x_{3}^{\prime}=x_{4}, \\
& x_{4}^{\prime}=\operatorname{Re} \operatorname{Pr} x_{4}-E c \operatorname{Pr} e^{-\varepsilon x_{3}} x_{2}^{2}-E c \operatorname{Pr}\left(x_{1}-1\right)^{2}
\end{aligned}
$$

subject to the following initial conditions;

$$
x_{1}(0)=0, x_{2}(0)=s_{1}, x_{3}(0)=0, x_{4}(0)=s_{2} .
$$

The unspecified initial conditions $s_{1}$ and $s_{2}$ in equation (21) are obtained iteratively using the Newton-Raphson algorithm together with fourth order Runge-Kutta integration scheme at a given terminal point. For a fixed set of parameter values, the accuracy of the missing initial conditions was checked by comparing the calculated value with the given value at the terminal point. The computations were done by a written program in MAPLE with a step size of $\Delta \eta=0.001$ selected to be satisfactory for a convergence criterion of $10^{-7}$ in nearly all cases.

\section{Results and Discussion}

In order to gain an insight into the dynamics of this physical problem, numerical computations for the representative velocity field, temperature field, skin friction, Nusselt number, entropy generation rate and Bejan number have been carried out by assigning some arbitrary chosen specific values to various thermophysical parameters controlling the flow system (see figures 2 -16). The Prandtl number ( $\mathrm{Pr})$ is assigned the values ranging from (Air) $0.71 \leq \operatorname{Pr} \leq 7.1$ (water) which are the most encountered fluids in nature and frequently used in engineering and industries. Accuracy of our numerical procedure in section 4 is validated by comparing the exact solution of the velocity field in equation (12) with our numerical results for the special case of constant fluid viscosity $(\varepsilon=0)$. The numerical results displayed in table 1 below are found to be in excellent agreement and hence attest the credibility of our results. 
Table 1: Computation showing comparison between the exact and numerical solution of velocity profile for $\mathrm{Re}=1, \mathrm{Ha}=1, \varepsilon=0$.

\begin{tabular}{|c|c|c|}
\hline$\eta$ & $\begin{array}{c}\text { Exact Solution } \\
w(\eta)\end{array}$ & $\begin{array}{c}\text { Numerical } \\
\text { Solution } w(\eta)\end{array}$ \\
\hline 0 & 0 & 0 \\
\hline 0.1 & 0.08812141 & 0.08812141 \\
\hline 0.2 & 0.17591234 & 0.17591234 \\
\hline 0.3 & 0.26426209 & 0.26426209 \\
\hline 0.4 & 0.35415939 & 0.35415939 \\
\hline 0.5 & 0.44671325 & 0.44671325 \\
\hline 0.6 & 0.54317727 & 0.54317727 \\
\hline 0.7 & 0.64497804 & 0.64497804 \\
\hline 0.8 & 0.75374838 & 0.75374838 \\
\hline 0.9 & 0.87136619 & 0.87136619 \\
\hline 1.0 & 1 & 1 \\
\hline
\end{tabular}

\subsection{Effects of parameter variations on velocity profiles}

Figures (2)-(3) show the effects of parameter variation on velocity profile. Generally, the axial velocity is zero at the fixed lower plate and gradually increases to its maximum value at the upper moving plate. An increase in magnetic field intensity (i.e. Ha increasing values) as showed in figure 2 causes a decline in the fluid motion towards the upper moving plate coupled with increasing fluid flow towards the lower fixed plate. Physically, this is justified because the application of transverse magnetic field always results in a resistive type of force called Lorentz force which is similar to drag force and tends to oppose the fluid motion toward the upper plate. However, the trend is opposite with increasing $R e$, leading to a rise in the fluid motion towards the upper moving plate due to increasing suction. It is interesting to note in figure 3 that an increase in the viscosity exponent $\varepsilon$ causes a rise in the fluid motion towards the upper moving plate as a results of decreasing viscosity. Since the fluid is lighter, it motion is enhanced by the uniform motion of the upper plate.

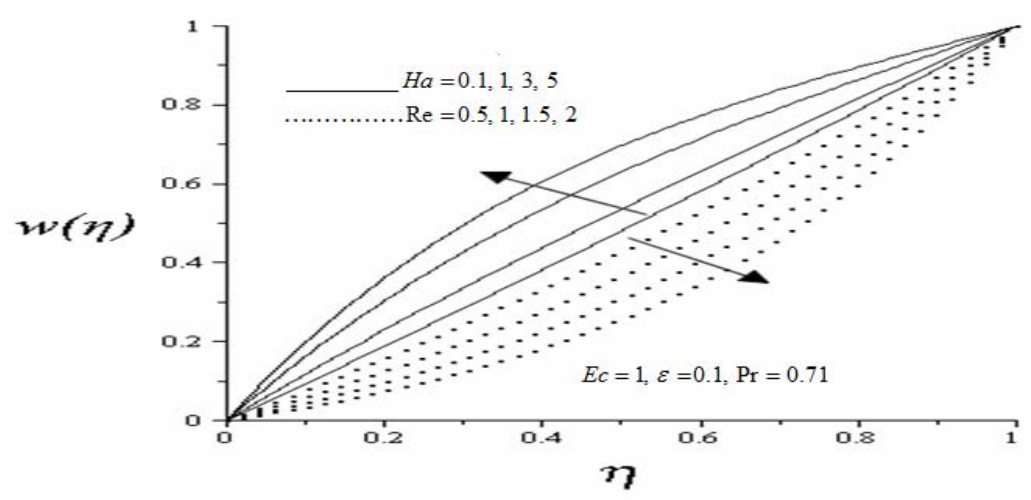

Figure 2: Effect of increasing Ha and Re on velocity profiles 


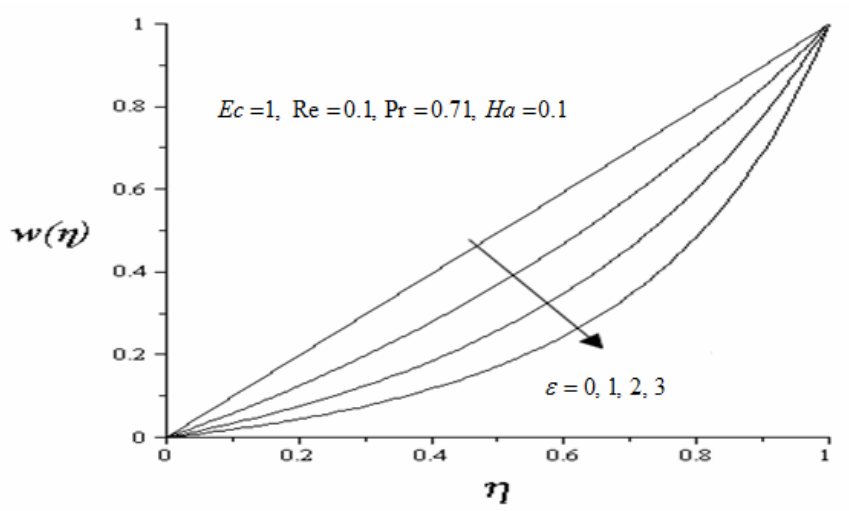

Figure 3: Effect of increasing $\varepsilon$ on velocity profiles

\subsection{Effects of parameter variations on temperature profiles}

Figures 4-6 represent the fluid temperature profiles across the channel. Generally, the fluid temperature is zero at the lower fixed plate and gradually increasing within the channel toward the upper moving plate. As $\mathrm{Ha}$ increases due to a rise in magnetic field intensity, a further increase in the fluid temperature is observed. This can be attributed to the increasing effect of Joule heating as shown in figure (4). Meanwhile, a fall in the fluid temperature is observed with an increase in $R e$ due to the combined effects of fluid injection/suction. As $R e$ increases, the temperature gradient at the upper moving plate increases. This in turn, increases the rate of heat loss due to suction, leading to a decrease in the fluid temperature. Figure (5) indicates fluid temperature elevation in the lower fixed plate region with an increase in $E c$ and $P r$. Increase in Ec increases the velocity gradient, leading to an increase in the viscous heating effect. Prandtl number defines the ratio of momentum diffusivity to thermal diffusivity for a given fluid implying that for lower $P r$ fluids (e.g. $P r=0.71$ corresponds to Air), heat diffuses faster away from the lower fixed plate and vice versa for higher Pr fluids (e.g. $\operatorname{Pr}=7.1$ corresponds to water). It is noteworthy that the rise in fluid temperature is more pronounced generally for high Prandtl number with temperature overshoot towards the upper moving plate due combined effect of a sharp decrease in temperature gradient and fluid suction. In figure (6), a rise in the fluid temperature towards the upper moving plate is observed for higher values of the viscosity exponent $\varepsilon$. This can be attributed to the fact that the fluid becomes lighter with enhance velocity gradient, leading to an increase in viscous heating.

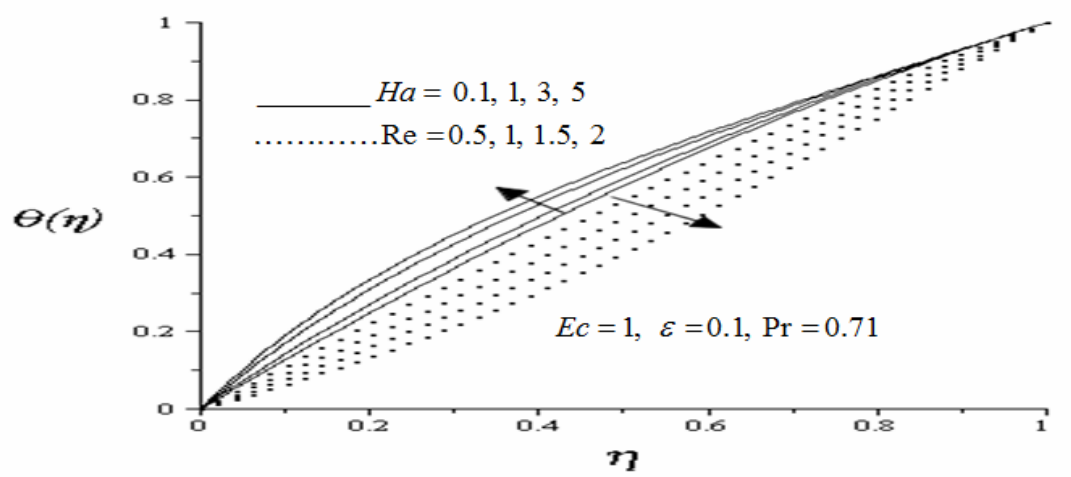

Figure 4: Effect of increasing Ha and Re on temperature profiles 


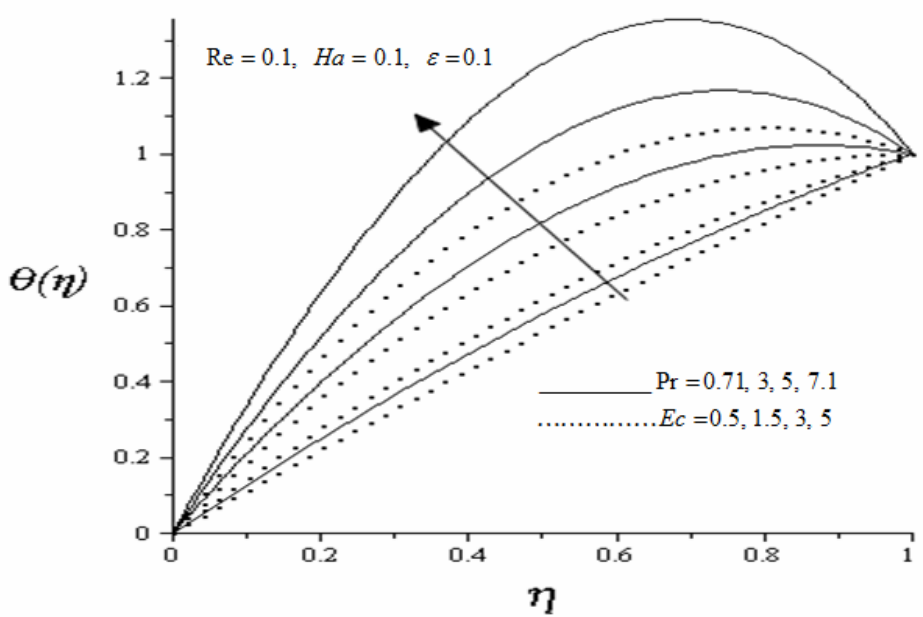

Figure 5: Effect of increasing Pr and Ec on temperature profiles

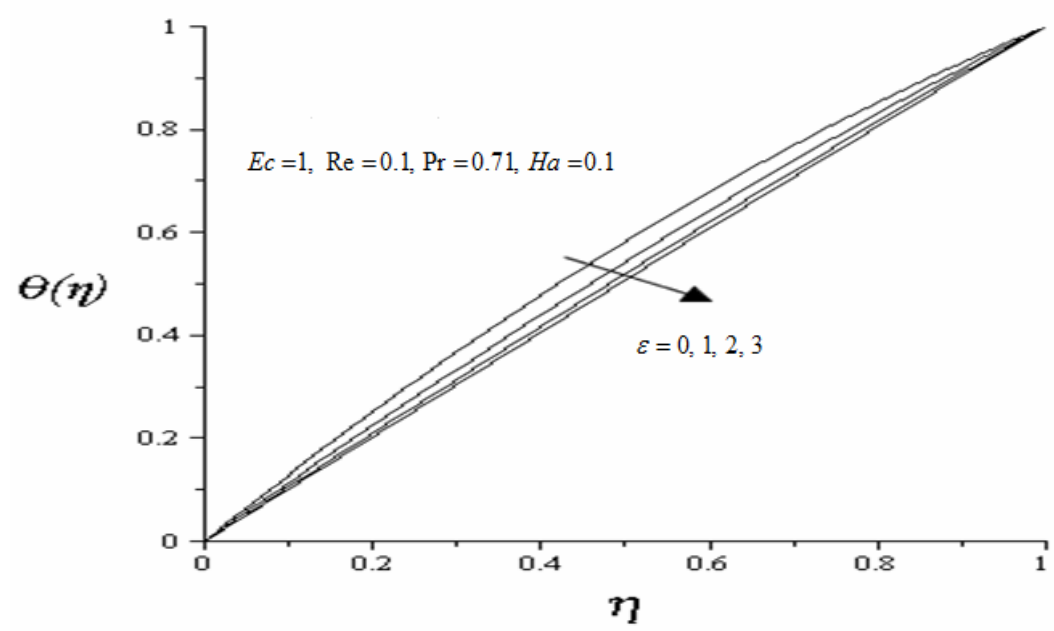

Figure 6: Effect of increasing $\varepsilon$ on temperature profiles

\subsection{Effects of parameter variations on skin friction and Nusselt number}

Figures (7)-(12) illustrate the effect of thermophysical parameters on skin friction and Nusselt number at the permeable plate surfaces. It is interesting to note in figure (7) that the skin friction at the lower fixed plate decreases with an increase in $R e$ and $\varepsilon$ but increases with an increase in $H a$. This is expected, since the fluid motion towards the lower plate decreases with increasing $R e$ and $\varepsilon$ due to injection and diminish fluid viscosity, consequently, the velocity gradient at the lower fixed plate decreases, leading to a fall in skin friction. Increases in $\mathrm{Ha}$ enhance the velocity gradient at the lower fixed plate due to magnetic field and Lorentz force, which in turn elevates the skin friction. The reversed situation is observed in figure (8) at the upper moving plate. The skin friction decreases with an elevation in the magnetic field intensity but increases with an increase in $\operatorname{Re}$ and $\varepsilon$. This may be attributed to the fact that a decrease in fluid viscosity enhances the velocity gradient at the upper moving plate while the Lorentz force due to magnetic field acts in opposite manner. It is 
noteworthy that suction at the upper moving plate tends to increase the skin friction whereas injection at the lower fixed acts in the opposite manner. The skin friction slightly decreases at both plate surfaces with an increase in Prandtl number (Pr) and Eckert number (Ec) as shown in figure (9). The temperature in the channel rises as $\mathrm{Pr}$ increases due to the effect of viscous dissipation. And the temperature rise could cause the decrease of viscosity and then reduces the skin friction. Figure (10) depicts the rate of heat transfer at lower plate surface. The Nusselt number $N u$ decreases with an increase in $R e$ and $\varepsilon$ but increases with an increase in $H a$. A decline in the fluid viscosity coupled with an increase in fluid injection enhances the temperature gradient while a rise in magnetic field intensity decreases the temperature gradient at the lower plate surface. In figure (11), elevation in the Nusselt number is observed with an increase in Re and $\varepsilon$ at the upper moving plate. This is expected, since temperature gradient at the upper moving plate increases due to a rise in fluid suction and a decline in fluid viscosity, consequently, the heat transfer rate increases. Increases in $\mathrm{Ha}$ in the presence of suction also enhance the temperature gradient slightly at the upper moving plate. Figure (12) shows that Nusselt number increase at the lower plate and decrease at upper moving plate with increasing Ec and Pr. It is interesting to note that at $E c=0.1$ the heat transfer rate at both plate surfaces is not affected with increasing value of $P r$.

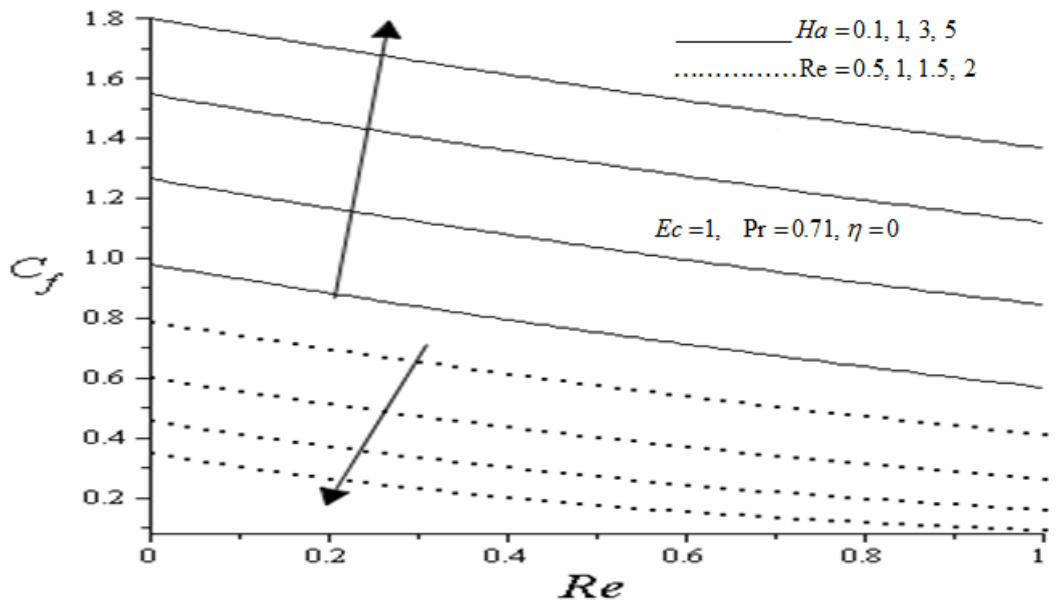

Figure 7: Skin friction at the lower plate with increasing Ha, Re, $\varepsilon$.

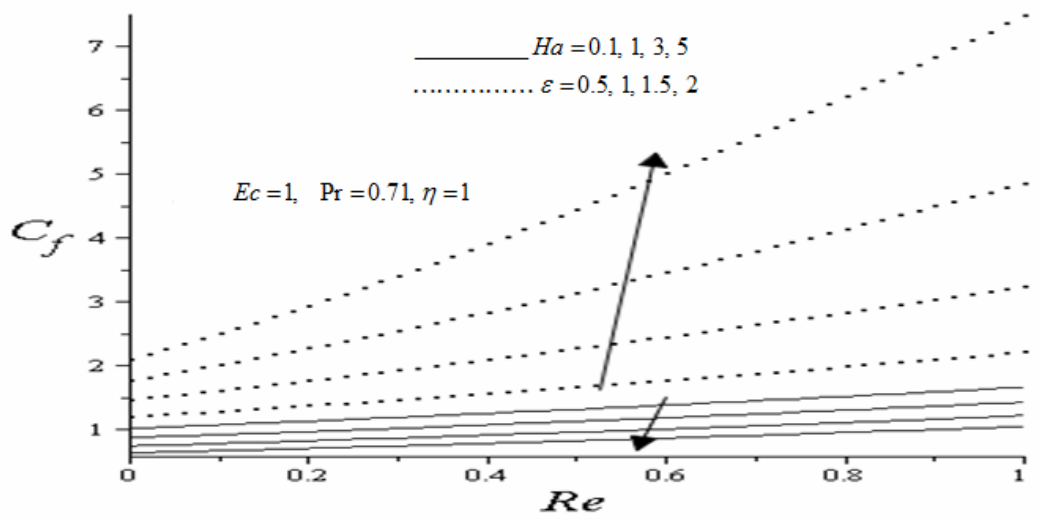

Figure 8: Skin friction at the upper plate with increasing $\mathrm{Ha}, \mathrm{Re}, \varepsilon$. 


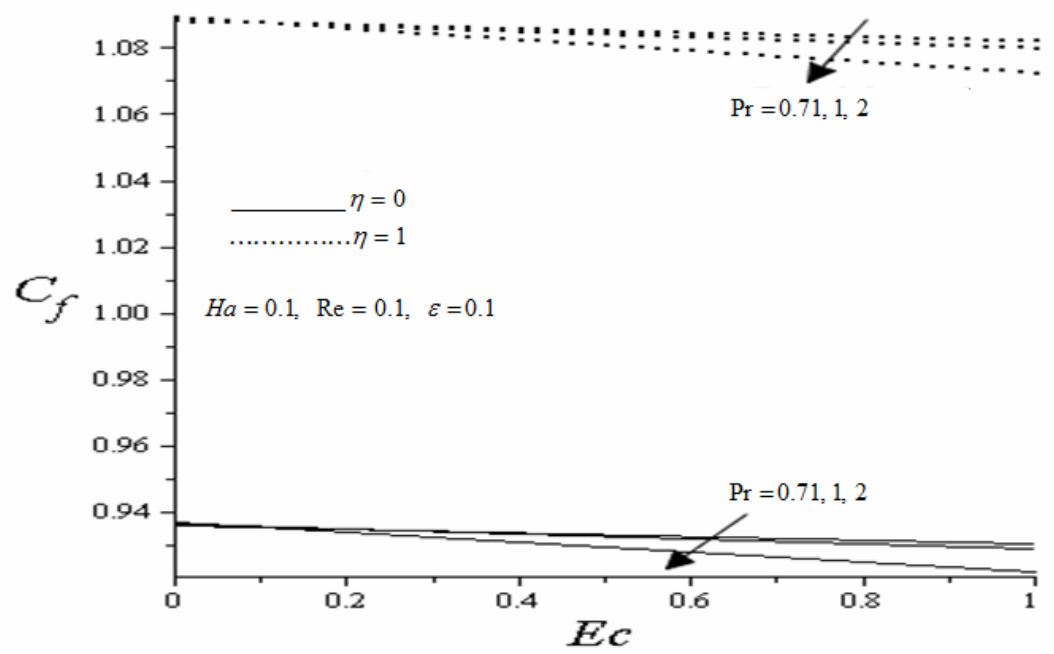

Figure 9: Skin friction with increasing $\operatorname{Pr}$, Ec.

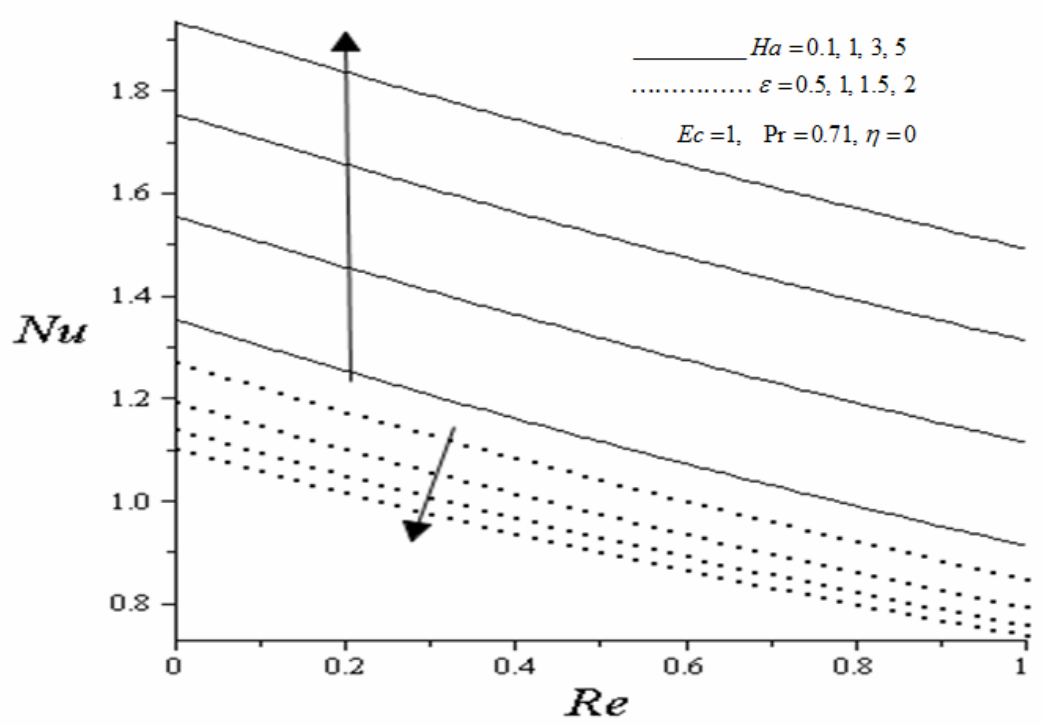

Figure 10: Nusselt number at the lower wall with increasing Ha, Re, $\varepsilon$.

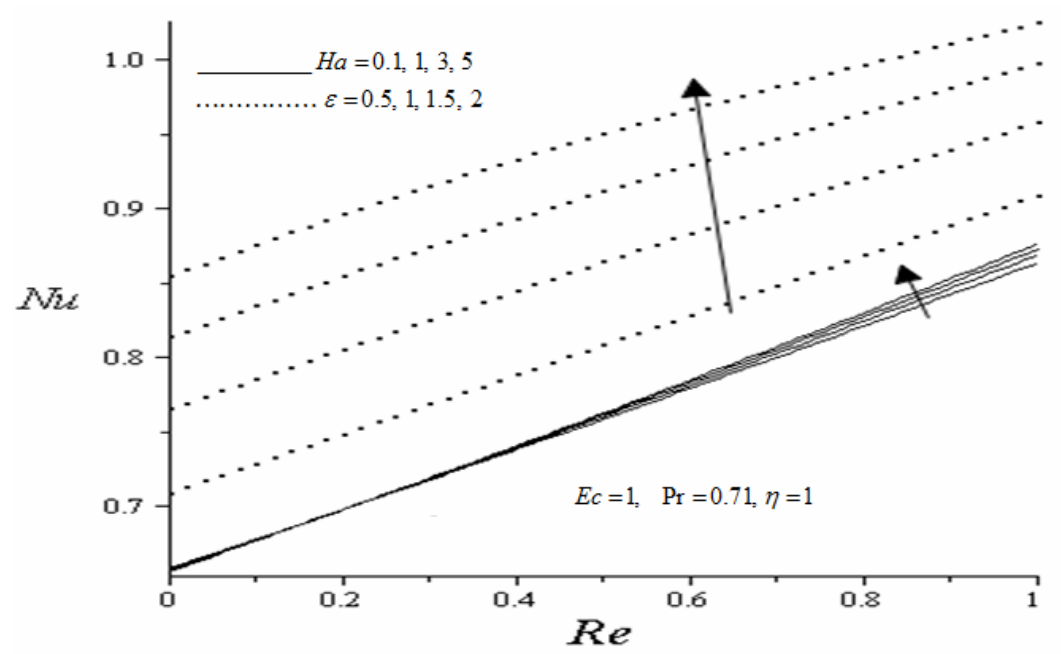

Figure 11: Nusselt number at the moving upper plate with increasing $\mathrm{Ha}, \mathrm{Re}, \varepsilon$. 


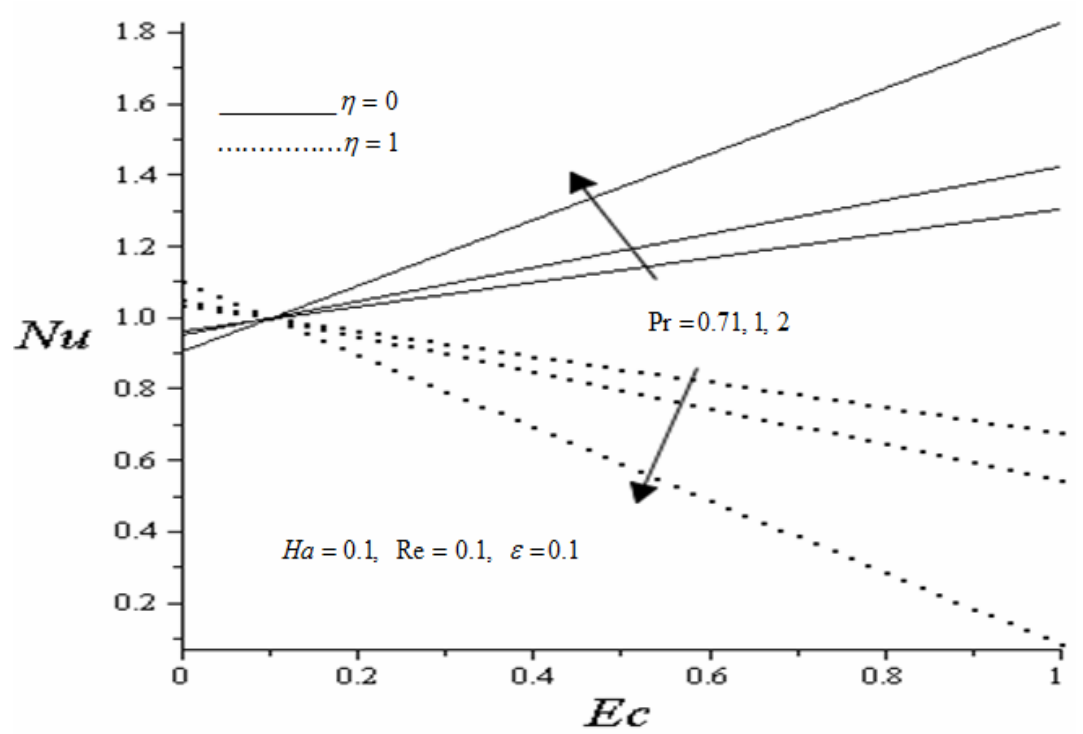

Figure 12: Nusselt number with increasing Pr, Ec.

\subsection{Effects of parameter variations on entropy generation rate}

Figures (13)-(14) show the influence of physical parameters on the local entropy generation number $N s$ plotted against $\eta$. The influence of the magnetic field parameter $H a$ and the injection/suction Reynolds number Re on the local entropy generation number is presented in figure (13). The presence of the magnetic field causes a decline in the entropy generation across the channel with more entropy generation at the lower fixed plate and less entropy production at the upper moving plate. Moreover, the trend is revised with an increasing Re. The local entropy generation is depressed at the lower fixed plate with increasing injection and enhanced at the upper moving plate with increasing suction. The effects of the group parameter $\mathrm{Br} \Omega^{-1}$ and viscosity exponent $\varepsilon$ on $N s$ are significant as it determines the relative importance of viscous effects. It is observed in figure (14) that an increase in group parameter causes the entropy production to increase. Entropy production is more at the lower fixed plate and less at the upper moving plate with increasing $\mathrm{Br} \Omega^{-1}$. The converse response is observed with less entropy generated at the lower fixed plate and more at the upper moving plate with increasing viscosity exponent $\varepsilon$ due to a decrease in fluid viscosity.

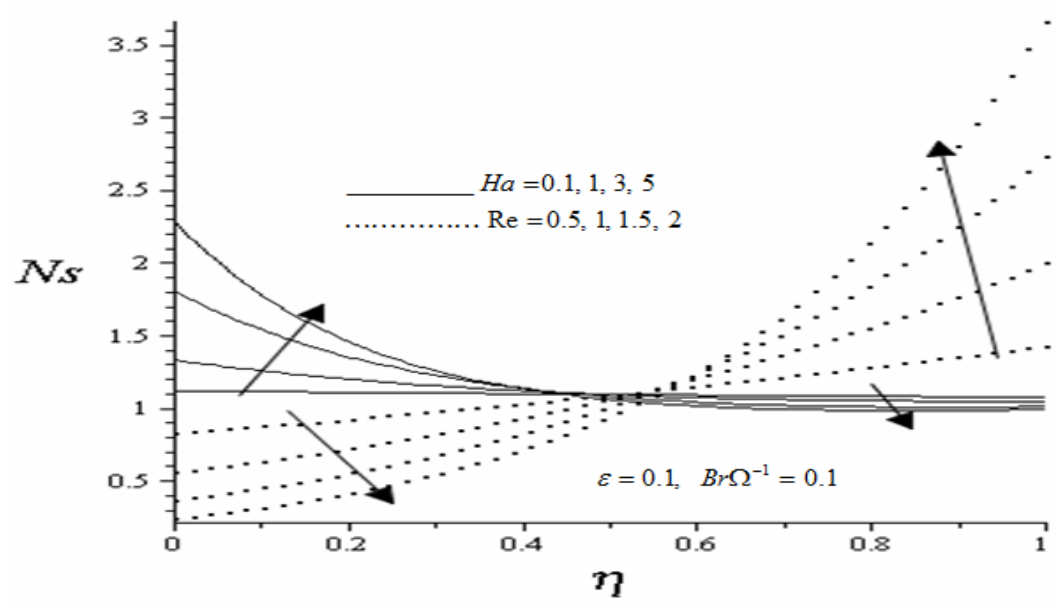

Figure 13: Effect of increasing Ha and Re on entropy generation rate. 


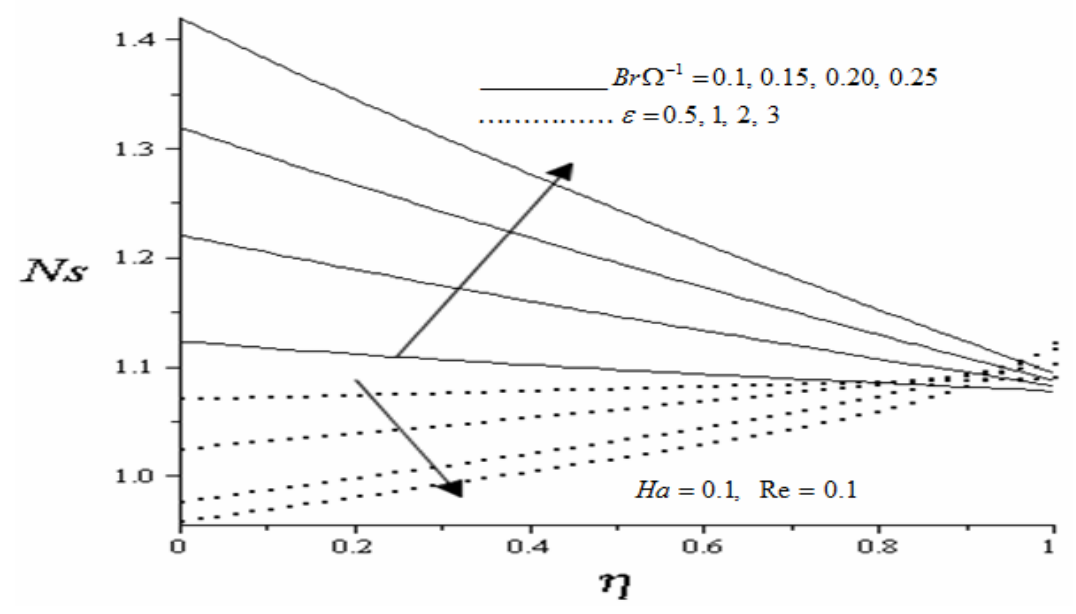

Figure 14: Effect of increasing $\mathrm{Br} \Omega^{-1}$ and $\varepsilon$ on entropy generation rate

\subsection{Effects of parameter variations on Bejan number}

To get an idea of whether the fluid friction irreversibility dominates over the heat transfer or vice versa, the Bejan number $B e$ is introduced. Figure (15) illustrates that with increase in the magnetic field parameter $\mathrm{Ha}$, the irreversibility effects due to fluid friction and magnetic field become dominant at the lower fixed plate region (with a decrease in $B e$ ) while the heat transfer irreversibility effects become dominant at the upper moving plate region (with an increase in $B e$ ). The situation is reversed with increasing injection/suction Reynolds number Re. The Bejan number increases at the lower fixed plate region due to a rise in injection and heat transfer irreversibility dominate. At the upper moving plate, the Bejan number decreases due to an increase in suction, consequently, viscous and magnetic field irreversibility become dominant. For the case of the group parameter $\mathrm{Br} \Omega^{-1}$ and viscosity exponent $\varepsilon$ as presented in figure (16), the fluid friction and magnetic field irreversibility strengthens across the channel with an increase in the group parameter. The irreversibility due to heat transfer is dominant with increasing viscosity exponent $\varepsilon$ due to a decrease in fluid viscosity.

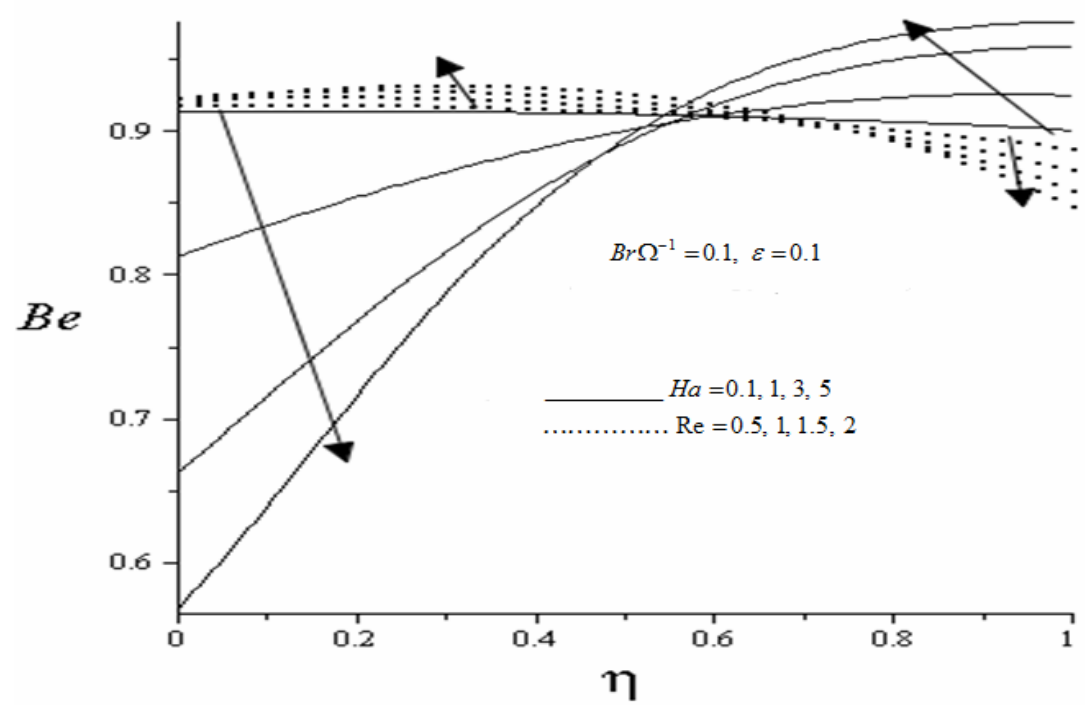

Figure 15: Effect of increasing $\mathrm{Ha}$ and $\mathrm{Re}$ on Bejan number 


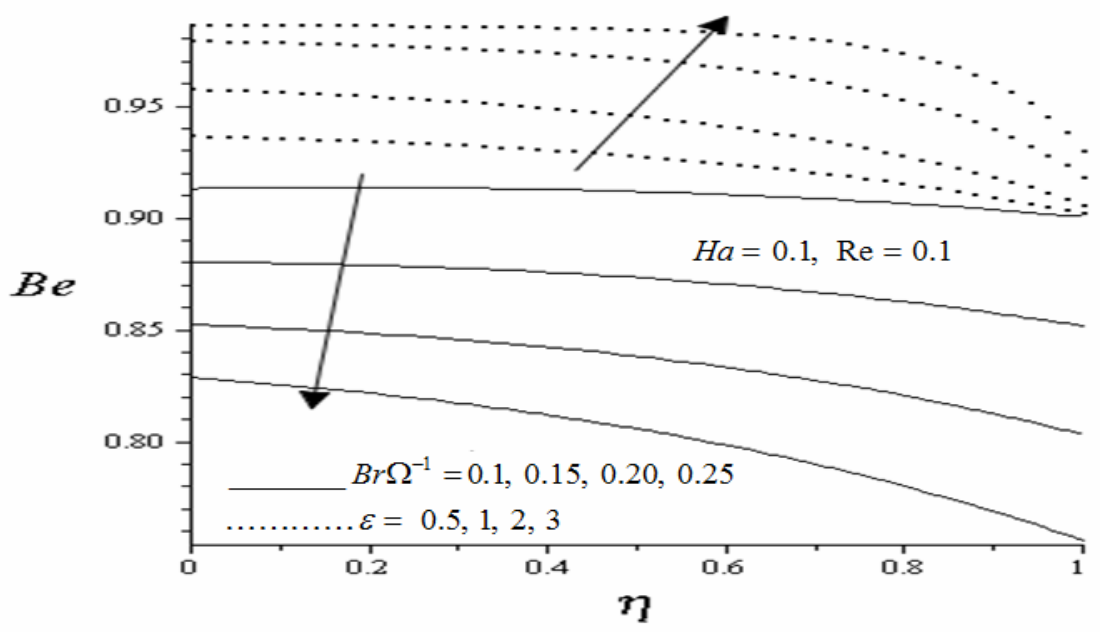

Figure 16: Effect of increasing $\varepsilon$ and $B r \Omega^{l}$ on Bejan number

\section{Conclusions}

This paper analyzed the inherent irreversibility in a variable viscosity hydromagnetic generalized Couette flow with suction/injection. Fourth-order Runge-Kutta-Fehlberg integration scheme together with shooting technique were employed to numerically solve the model governing equations. Some of the results obtained can be summarized as follows:

- Increase in Re, $\varepsilon$ increases fluid motion towards the upper moving plate while increase in Ha increases fluid motion towards the lower fixed plate.

- Fluid temperature at the lower fixed plate region increases with Ha, Ec, $\mathrm{Pr}$ while increase in Re, $\varepsilon$ increases the temperature at the upper moving plate region.

- Skin friction at the upper moving plate is higher than that at the lower fixed plate. Skin friction increases with $\mathrm{Ha}$ but decreases with $R e$ and $\varepsilon$ at the lower fixed plate. The situation is reversed at the upper moving plate.

- Nusselt number increases with $H a, R e, \varepsilon$ and decreases with Ec and Pr at the upper moving plate. At the lower fixed plate the $\mathrm{Nu}$ decreases with $\mathrm{Re}, \varepsilon$, and increases with $\mathrm{Ha}, \mathrm{Pr}, \mathrm{Ec}$.

- Local entropy generation rate increases with $\mathrm{Br} \Omega^{-1}$ but decreases with $\varepsilon$. Increase in Ha decreases entropy production at the moving upper plate while increase in Re decreases entropy generation at the lower fixed plate.

- A decrease in fluid viscosity increases Bejan number while an increase group parameter $\mathrm{Br} \Omega^{-1}$ decreases Bejan number. Increase in $R e$ increases $B e$ at the lower fixed plate and decreases $B e$ at the upper moving plate. The situation is reversed with increasing $\mathrm{Ha}$.

From the following observations, it is concluded that the optimal design and the efficient performance of a flow system or a thermally designed system can be improved by choosing the appropriate values of the physical parameters. 


\section{Acknowledgement}

The authors gratefully acknowledge the financial support of South African National Research Foundation and the valuable suggestions of the anonymous reviewers.

\section{References}

[1] Moreau R., Magnetohydrodynamics, Kluwer Academic Publishers, Dordrecht, 1990.

[2] Agarwal J. P., On generalized Couette flow in hydromagnetics. Appl. Scient. Res. 9 B, 255-265, 1962.

[3] Soundalgekar V.H., On generalized MHD Couette flow with heat transfer. Proc. Indian Acad. Sci., 64, 304-314, 1966.

[4] Sutton G.W., Sherman A., Engineering magnetohydromagnetics. McGraw-Hill Book Co. Inc., New York, 1965.

[5] Attia H.A, Kotb N.A, MHD flow between parallel plates with heat transfer, Acta Mechanica 117, 215-220, 1996.

[6] Ram P., Bhandari A, Sharma K., Effect of magnetic field-dependent viscosity on revolving ferrofluid, Journal of Magnetism and Magnetic Materials 322 (21), 3476-3480, 2010.

[7] Makinde O. D., Onyejekwe O. O., A numerical study of MHD generalized Couette flow and heat transfer with variable viscosity and electrical conductivity. Journal of Magnetism and Magnetic Materials 323, 2757-2763, 2011.

[8] Bejan A., Second-Law analysis in heat transfer and thermal design. Adv. Heat Transfer 15, 1-58, 1982.

[9] Mahmud S., Fraser R.A., Flow, thermal and entropy generation characteristic inside a porous channel with viscous dissipation. Int. J. Therm. Sci., 44, 21-32, 2005.

[10] Aziz A., Entropy generation in pressure gradient assisted Couette flow with different thermal boundary conditions. Entropy, 8(2), 50-62, 2006.

[11] Eegunjobi A.S., Makinde O.D., Effects of Navier slip on entropy generation in a porous channel with suction/injection. Journal of Thermal Science and Technology, 7(4), $522-535,2012$.

[12]Shojaefaed M.H., Noorpoor A.R., Avanesians A., and Ghaffapour M. Numerical investigation of flow control by suction and injection on a subsonic airfoil. Am .J. Appl. Sci 20(10),1474-1480, 2005.

[13]Makinde O.D., Eegunjobi A.S., Effects of convective heating on entropy generation rate in a channel with permeable walls. Entropy 15, 220-233, 2013.

[14] Wood L.C., Thermodynamics of Fluid Systems, Oxford University Press, Oxford, 1975.

[15]Tasnim S.M., Mahmud S., Mamum M.A.H., Entropy generation in a porous channel with hydromagetic effect. Int. J. Exergy, 3, 300-308, 2002.

[16] Klemp K., Herwig H., Selmann M., Entrance flow in channel with temperature dependent viscosity including viscous dissipation effects. Proc. Third Int. Cong. Fluid Mech., Cairo, Egypt, 3, p. 1257, 1990.

$[17] \mathrm{Na}$ T.Y., Computational methods in engineering boundary value problems, Academic press, New York, 1979. 